\section{Surface Functionalization Achieved by Photo-Initiated Thiol-Ene Chemistry}

Strategies to modify materials and surfaces have become important for a wide variety of disciplines, including bioengineering, photovoltaics, and heterogeneous catalysis. These strategies are especially attractive for biological applications, where tailored surfaces are used for cell substrates, detection platforms, and binding assays. Two commonly used substrates for biological studies are silica and glass, both of which can be modified through siloxane chemistry. Now, A. Bertin and H. Schlaad of the Max Planck Institute of Colloids and Interfaces in Potsdam, Germany, have offered a new strategy describing the use of thiol-ene photochemistry to create polymeric and organic coatings for glass.

As reported in the December 22, 2009 issue of Chemistry of Materials (DOI: $10.1021 / \mathrm{cm} 902176 n$; p. 5698), Bertin and Schlaad used surface-bound thiols to initiate radical polymerizations and to add to terminal alkene moieties. Glass slides were first modified with (3-mercaptopropyl)trimethoxylsilane, the source of thiols. The initial demonstration involved surfaceinitiated radical polymerization of methacrylic acid (MAA). By exposing an aqueous solution of MAA to UV light for $24 \mathrm{~h}$ at room temperature, the resulting hybrid surface contained a $200 \mathrm{~nm}$ coating of poly(MAA). The presence of the surfacegrafted poly(MAA) caused a decrease in the contact angle from $80^{\circ}$ (glass-thiol surface) to $52^{\circ}$ (polymer-modified glass).

Alkenes can also be immobilized to thiolmodified glass with this technique. By exposing thiol-functionalized glass slides to solutions of 1-allyl- $\alpha$-D-glucopyranoside in 1:1 THF/methanol (v/v), $1 H, 1 H, 2 H$ perfluoro-1-decene in THF, or poly(butadiene) in THF to UV light for $24 \mathrm{~h}$ at room temperature, hybrid surfaces were designed that allowed for the modulation of surface properties. The resulting contact angles were $66^{\circ}, 90^{\circ}$, and $87^{\circ}$ for the glucose-, perfluoroalkane-, and poly(butadi- ene)-modified glass, respectively. In order to further explore the versatility of this photochemical strategy, the activity of the glucose-modified glass was determined. By exposing glass slides that present carbohydrates to a solution of fluorescent Concanavalin A (ConA), the interaction between the sugar units and the lectin could be monitored by fluorescence microscopy. The researchers determined that the inclusion of a flexible polymer layer-poly(butadiene)-allowed for a higher degree of binding of ConA.

According to Bertin and Schlaad, the ease with which thiols can be added to glass and silica makes this UV-mediated immobilization an attractive option for biointerfacial surfaces. By exploring the utility of this technique, the researchers want to develop platforms for carbohydrate microarrays, biosensors, and other bioassays.

DEVIN G. BARRETT
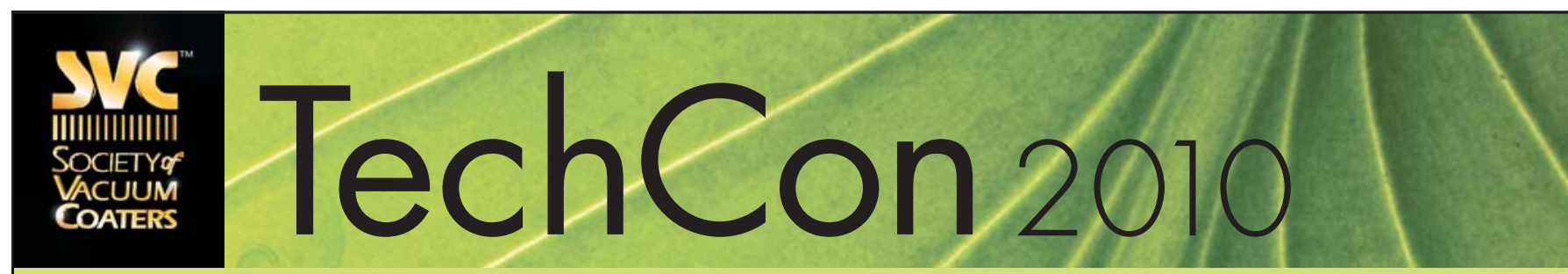

April 17-22, 2010 - Orlando World Center Marriott Resort and Convention Center - Orlando, FL

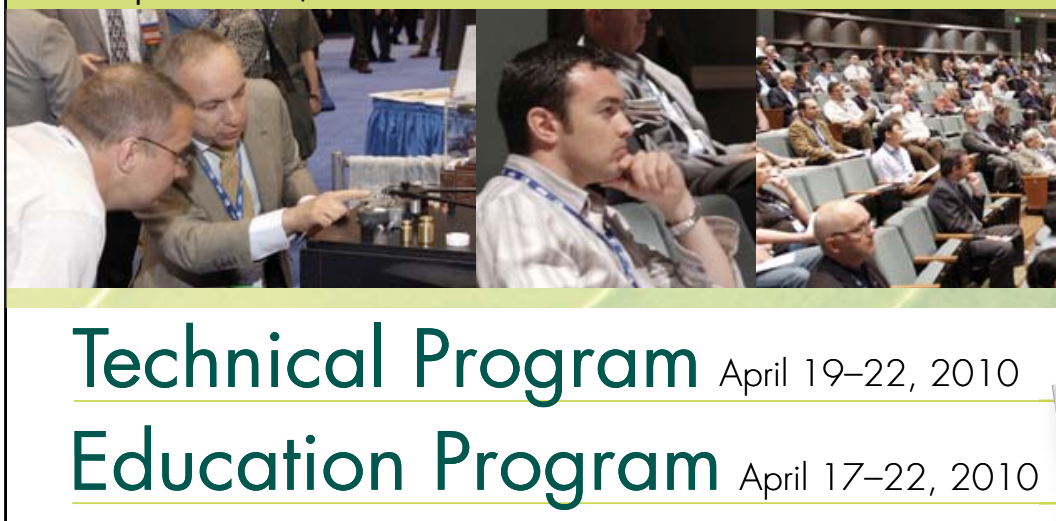

Exhibit April 20-21, 2010

For information on the 53rd Annual

Technical Conference, visit us on-line at

\title{
WWW.svC.org
}

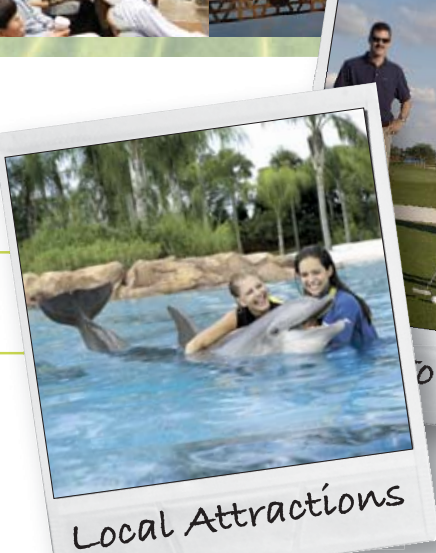

(n) 